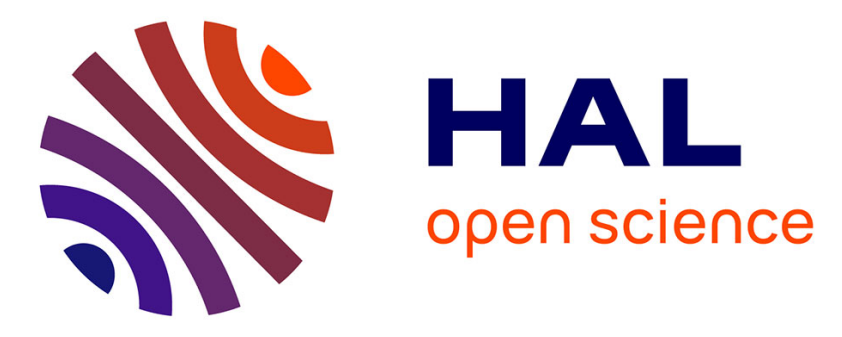

\title{
Geometrical deviations versus smoothness in 5-axis high-speed flank milling
}

Pierre-Yves Péchard, Christophe Tournier, Claire Lartigue, Jean-Pierre

Lugarini

\section{- To cite this version:}

Pierre-Yves Péchard, Christophe Tournier, Claire Lartigue, Jean-Pierre Lugarini. Geometrical deviations versus smoothness in 5-axis high-speed flank milling. International Journal of Machine Tools \& Manufacture, 2009, 49 (6), pp.454-461. 10.1016/j.ijmachtools.2009.01.005 . hal-00572764

\section{HAL Id: hal-00572764 \\ https://hal.science/hal-00572764}

Submitted on 2 Mar 2011

HAL is a multi-disciplinary open access archive for the deposit and dissemination of scientific research documents, whether they are published or not. The documents may come from teaching and research institutions in France or abroad, or from public or private research centers.
L'archive ouverte pluridisciplinaire HAL, est destinée au dépôt et à la diffusion de documents scientifiques de niveau recherche, publiés ou non, émanant des établissements d'enseignement et de recherche français ou étrangers, des laboratoires publics ou privés. 


\section{Author's Accepted Manuscript}

Geometrical deviations versus smoothness in 5-axis high-speed flank milling

Pierre-Yves Pechard, Christophe Tournier, Claire Lartigue, Jean-Pierre Lugarini

PII: $\quad$ S0890-6955(09)00012-1

DOI: doi:10.1016/j.ijmachtools.2009.01.005

Reference: $\quad$ MTM2378

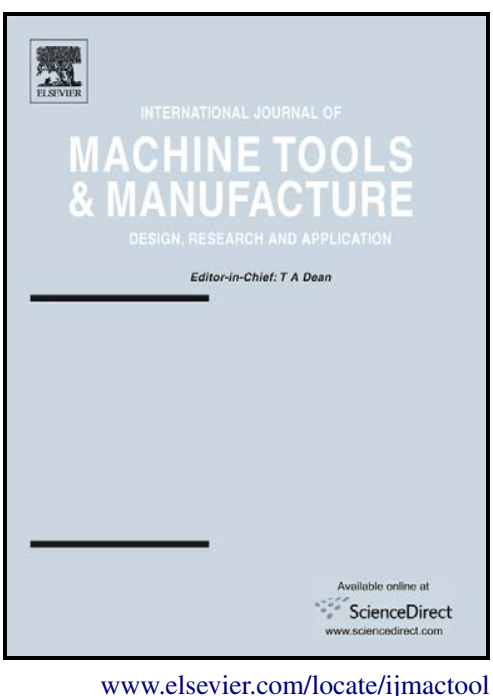

To appear in: International Journal of Machine Tools \& Manufacture

Received date: 14 September 2008

Revised date: 2 January 2009

Accepted date: 14 January 2009

Cite this article as: Pierre-Yves Pechard, Christophe Tournier, Claire Lartigue and Jean-Pierre Lugarini, Geometrical deviations versus smoothness in 5-axis highspeed flank milling, International Journal of Machine Tools \& Manufacture (2009), doi:10.1016/j.ijmachtools.2009.01.005

This is a PDF file of an unedited manuscript that has been accepted for publication. As a service to our customers we are providing this early version of the manuscript. The manuscript will undergo copyediting, typesetting, and review of the resulting galley proof before it is published in its final citable form. Please note that during the production process errors may be discovered which could affect the content, and all legal disclaimers that apply to the journal pertain. 


\section{Geometrical deviations versus smoothness in 5-axis high-speed flank milling}

Pierre-Yves Pechard, ${ }^{\text {a,c }}$ Christophe Tournier, ${ }^{\text {a,* }}$ Claire Lartigue, ${ }^{\mathrm{a}, \mathrm{b}}$ Jean-Pierre Lugarini. ${ }^{\mathrm{c}}$ ${ }^{a}$ LURPA, ENS Cachan, Université Paris Sud 11 61 av du pdt Wilson, 94235 Cachan, France

${ }^{\mathrm{b}}$ IUT de Cachan, Université Paris Sud 11 9 avenue de la division Leclerc, 94234 Cachan, France

${ }^{\mathrm{c}}$ Missler Software, 7 Rue du Bois Sauvage, 91055 Evry, France

* tournier@lurpa.ens-cachan.fr, Tel: 33147402 996, Fax: 33147402211

Preprint submitted to Elsevier 


\title{
Geometrical deviations versus smoothness in 5-axis high-speed flank milling
}

\begin{abstract}
The paper deals with the Generation of Optimized 5-aXis Flank milling trajectories. Within the context of 5-axis High-Speed Machining, oscillatory trajectories may penalize process efficiency. The control of the trajectory smoothness is as essential as the control of geometrical deviations. For this purpose the Geo5XF method based on the surface representation of the tool trajectory has been developed. In flank milling, this surface, also called the Machining Surface (MS), is the ruled surface locus of the tool axes defining the trajectory. Based on a first positioning, the method aims at globally minimizing geometrical deviations between the envelope surface of the tool movement and the designed surface by deforming the MS while preserving trajectory smoothness. The energy of deformation of the MS is used as an indicator of the smoothness. Hence, in most cases, results obtained using Geo5XF show that minimum energy tool paths lead to minimal machining time. As geometrical deviations are not minimized for minimum energy tool paths, a compromise must be reached to find the best solution.
\end{abstract}

Key words: Flank milling, minimum energy, High Speed Milling, Singular Value Decomposition, geometrical deviations

Preprint submitted to Elsevier

22 January 2009 
- $M S(u, v)$ Machining Aurface

- $C b(u)$ Bottom curve

- $C t(u)$ Top curve

- $C b_{i}$ Control point of the bottom curve

- $C t_{i}$ Control point of the top curve

- $N_{i m}(u)$ B-spline basis functions

- $n_{1}$ number of control points of the bottom curve

- $n_{2}$ number of control points of the top curve

- $S(u, v)$ Design Surface

- $A_{k}(u)$ Sampled points on the tool axis

- $B_{k}(u)$ Projected points on the Design Surface

- $B_{k}^{p}(u)$ Projection of $B_{k}(u)$ point on the tool axis

- $C_{k}(u)$ Intersection points on the tool Surface

- $\xi_{k}(u)$ Initial deviation

- $\vec{n}_{k}(u)$ Normal vector to the Machining Surface

- $h(u)$ Distance between $C b(u)$ and $C t(u)$

- $k_{p}(u)$ Distance between $C b(u)$ and $B_{k}^{p}(u)$

- $\vec{D}_{C_{k}(u)}$ Displacement of point $C_{k}(u)$

- $\vec{D}_{B_{k}^{p}(u)}$ Displacement of point $B_{k}^{p}(u)$

- $e_{k}(u)$ Optimized deviation

- $\lambda_{i}$ Eigenvalues

- $W$ Sum of squared deviations

- E Energy of deformation of the Machining Surface

- $i, j, k$ cosine of the tool axis orientation

- $A, C$ Rotary axes of the machine tool 
By promoting 5-axis HSM and the use of integrated CAD/CAM/CNC systems, a significant improvement in machining efficiency and accuracy has been achieved. Due to many advantages, such as a high removal material rate or a better surface roughness [1], flank milling has become very popular compared with point milling. In particular, this process is now widely used for the machining of slender complex parts like impellers or turbine blades. However, process advantages now motivate industrials to apply high-speed flank milling to any types of surfaces, leading up to new challenges for tool path calculation. Over the last decade, several positioning strategies for flank milling have been developed with the objective of minimizing geometrical deviations [2]. Although the local control of geometrical deviations is still a major issue, the smoothness of the calculated tool trajectory is essential to ensure high performance machining. Indeed, oscillatory trajectories may penalize process efficiency.

Methods proposed in literature aim at developing positioning strategies so that geometrical deviations between the machined surface and the designed surface are minimized. Most flank milling methods rely on a first tool positioning on the surface at one or more points, followed by the tool positioning optimization to reduce geometrical errors [3]. Over the years, methods have evolved by increasing the number of contact points with the objective of minimizing the geometrical deviations to the detriment of trajectory smoothness and computation time.

Lui [4] developed two methods to machine non-developable ruled surfaces with 
hemispherical tools. The first one, the Simple Point Offset (SPO), consists

in positioning the tool collinear to the surface rule passing through a point offset at the mid curve. Geometric errors are thus divided into overcuts and undercuts. The second method is the Double Point Offset (DPO) method. Two contact points located at the parametric values 0.25 and 0.75 of the rule, are offset by a value equal to the tool radius along the normal to the surface. These two offset points allow to define the tool axis orientation.

Redonnet et al., [5] proposed a positioning strategy based on three contact points. The axis of a cylindrical tool is initially positioned collinear to the rule by an offset distance value equal to the tool radius. A rotation around the normal at the surface at the mid-curve is applied so that the tool becomes tangent to the two directrices while preserving a contact point with a rule. This leads to a system of seven transcendental equations that must be solved. An extension of the method to conical tools was developed in [6]. More recently, the authors proposed an improvement of the method considering that the rotation axis is defined as one of the variables of the seven equations and the contact point is located at the mid-curve [7].

The positioning method developed by Bedi et al, [8] consists in positioning the tool tangent to the two boundary curves of the ruled surface. As contact points are limited to the boundaries, the maximum error is located at the mid-curve. To reduce such errors, an optimization is developed in [3]. Contact points are moved along the rule until the deviation between the rule and the tool is minimized. A last step is thus carried out by moving contact points in the transverse direction until a third contact point is obtained, decreasing further geometrical errors. 
the tool movement and the surface to be machined are all positive.

Gong developed the Three Point Offset method (TPO) for flank milling using a cylindrical tool [10]. Each tool axis is positioned thanks to three contact curves belonging to the offset surface of the surface to be machined. The initial position is defined by two points belonging to the boundary curves. One of these points is moved until the deviation between the tool axis and the third curve is less than a threshold. A surface is built by interpolating the tool extremities with B-spline curves. The surface is thereafter deformed so that the geometrical deviations between the machining surface and the offset surface are minimized.

In previous works, a surface-based approach for tool positioning in flank milling has been proposed in [11]. The method relies on a first positioning of the tool according to a two contact point method. Extremity points of the tool axes are approximated by two curves. These two curves define the directrices of a ruled surface, defined as the Machining Surface $(M S)$. To improve tool positioning, the Machining Surface is deformed so that the geometrical deviations between the envelope surface of the tool movement and the designed surface are minimized according to the least-square criterion. The deformation is performed thanks to the displacement of the curve control points using the small displacement torsor method [12]. This leads to solve a large linear system. First results have shown the benefit of using the surface-based approach to improve tool positioning [11]. The control of the geometrical deviations is as essential as the smoothness of the calculated tool trajectory to ensure high performance machining. Indeed, works have shown that efficiency in 5-axis high-speed machining is better when trajectories are smooth [13], [14]. The 
ness of the trajectory can be apprehended through the energy; a curve which minimizes its energy then minimizes its curvature variations. As a result a minimum energy trajectory minimizes curvature variations and consequently acceleration and jerk variations are decreased during trajectory processing. Taking advantage of the surface representation of the trajectory, the smoothness is evaluated by calculating the energy of the surface. Therefore, the issue of minimizing geometrical deviations between the envelope surface of the tool movement and the designed surface coupled to the trajectory smoothing in 5-axis flank milling is investigated in this paper. The method relies on the concept of the $M S$ which has already shown its benefit not only for optimized tool positioning as regards geometrical deviations, but also for general 5-axis milling optimization to answer kinematical criteria [15].

In the next section, the four steps of the method for optimized 5-axis flank milling trajectories are detailed. In particular, specific attention is paid to the calculation of the geometrical deviations between the envelope surface of the tool movement and the designed surface. The deformation of the initial tool positioning, which is expressed as a surface, leads to solve a large linear system. As the system is generally ill-conditioned, it is solved by means of the SVD giving thus approximate solutions. In section 3, the energy of deformation of the surface trajectory is introduced as an indicator of the trajectory smoothness. For each estimate of the solution, the cumulated energy during machining is calculated. A series of machining tests are carried out in section 4 , highlighting that the minimum energy tool trajectory is in general the minimum machining time trajectory. Results demonstrate the efficiency of the surface-based approach for high performance process. 
2 Generation of Optimized Flank milling trajectories

The method for Generation of Optimized 5-aXis Flank milling trajectories (Geo5XF) is an extension of the method developed in [11] and consists of four main steps: Initial tool positioning; Approximation of the tool axis extremities by two curves defining the two directrices of the $M S$; Calculation of the deviations between the envelope surface and the designed surface; Positioning optimization by deforming the $M S$ (Fig.1).

\subsection{Initial positioning}

The initial tool positioning is carried out using the SPO positioning method developed by Liu [4] for its robustness and simplicity to implement. Indeed, whatever the nature of the surface to be machined, this method provides a result which is always exploitable in a minimal computation time. At this stage, a set of tool axes is created defining the initial tool positioning for which the values of undercuts and overcuts are generally too large.

\subsection{Directrix definition}

Cubic B-spline curves are then associated to the extremity points of the set of axes according to the least-square criterion. These two curves define the directrices of the ruled parametric surface $M S(u, v)$ defining the Machining Surface (Fig.1), the equation of which is:

$$
M S(u, v)=(1-v) \cdot C b(u)+v \cdot C t(u)
$$


bottom curve and the top curve respectively. These curves are calculated as follows:

$$
\overrightarrow{C b}(u)=\sum_{l=1}^{n_{1}} N_{l 3}(u) \overrightarrow{C b}_{l} \quad \text { and } \quad \overrightarrow{C t}(u)=\sum_{m=1}^{n_{2}} N_{m 3}(u) \overrightarrow{C t}_{m}
$$

where $N_{l 3}(u)$ and $N_{m 3}(u)$ are the basic spline functions as defined in [16].

\subsection{Calculation of the geometrical deviations}

In the literature, calculation methods of the geometrical deviations between the designed surface and the machined surface are most often based on the envelope surface of the tool movement. More generally, the calculation method is directly linked to the method used for tool path generation [8], [9], [11], [17], [18].

Concerning the envelope surface calculation, some methods use the implicit equations of the designed surface to determine the exact representation of the envelope surface [17], [18]. Nevertheless, for simplicity reasons, kinematical methods leading to an approximation of the envelope surface are used in [8], [9], [11]. In [19], the authors compared the main methods of geometrical error calculation proposed in literature and showed that kinematical methods relying on double projection provide good results regarding effective geometrical errors. Therefore, the kinematical approach as developed in [11] relying on a double projection method is adopted in this study.

The envelope surface is approximated by considering static instances of the tool axis movement. To each static instance, defined by a value $u^{*}$ of the $u$ 
(Fig.2). Each point $A_{k}\left(u^{*}\right)$ is normally projected onto the designed surface, giving the point $B_{k}\left(u^{*}\right)$ which is in turn projected onto the considered tool axis, giving the point $B_{k}^{p}\left(u^{*}\right)$. This last projection intersects the tool surface at a point $C_{k}\left(u^{*}\right)$. Therefore, the geometrical deviation $\xi_{k}\left(u^{*}\right)$, between the envelope surface and the designed surface at the point $B_{k}\left(u^{*}\right)$ is given by:

$$
\xi_{k}\left(u^{*}\right)=\overrightarrow{C_{k}\left(u^{*}\right) B_{k}\left(u^{*}\right)} \cdot \vec{n}_{k}\left(u^{*}\right)
$$

where $\vec{n}_{k}\left(u^{*}\right)$ is the normal to the machining surface $M S(u, v)$ at $C_{k}\left(u^{*}\right)$, and $h\left(u^{*}\right)$ is the height of the tool at $u^{*}$ and $k_{p}\left(u^{*}\right)$ is the distance $\left\|\overrightarrow{C b\left(u^{*}\right) B_{k}^{p}\left(u^{*}\right)}\right\|$.

\subsection{Optimization through the MS deformation}

The optimization is carried out by applying a deformation to the $M S$ so that the envelope surface of the tool movement best fits the designed surface. The surface deformation is obtained by applying a small displacement $\vec{D}_{C_{k}\left(u^{*}\right)}$ to each sampled point of the tool surface, giving the optimized deviation[12] (Fig.3):

$$
e_{k}\left(u^{*}\right)=\xi_{k}\left(u^{*}\right)-\vec{D}_{C_{k}\left(u^{*}\right)} \cdot \vec{n}_{k}\left(u^{*}\right)
$$

However, considering that displacements are small, the projection of the small displacement of $C_{k}\left(u^{*}\right)$ onto the normal to the tool surface $\vec{n}_{k}\left(u^{*}\right)$ is assumed to be equal to the projection of the small displacement of $B_{k}^{p}\left(u^{*}\right)$ onto $\vec{n}_{k}\left(u^{*}\right)$ :

$$
\vec{D}_{C_{k}\left(u^{*}\right)} \cdot \vec{n}_{k}\left(u^{*}\right)=\vec{D}_{B_{k}^{p}\left(u^{*}\right)} \cdot \vec{n}_{k}\left(u^{*}\right)
$$


Hence, by expressing $\vec{D}_{B_{k}^{p}\left(u^{*}\right)}$ in function of the displacement of the control points of each directrix, this yields to:

$$
\begin{aligned}
e_{k}\left(u^{*}\right)=\xi_{k}\left(u^{*}\right) & -\left(1-\frac{k_{p}\left(u^{*}\right)}{h\left(u^{*}\right)}\right) \cdot \sum_{l=0}^{n 1}\left[N_{l 3}\left(u^{*}\right) \cdot \overrightarrow{\delta C b_{l}}\right] \cdot \vec{n}_{k}\left(u^{*}\right) \\
& +\left(\frac{k_{p}\left(u^{*}\right)}{h\left(u^{*}\right)}\right) \cdot \sum_{m=0}^{n 2}\left[N_{m 3}\left(u^{*}\right) \cdot \overrightarrow{\delta C t_{m}}\right] \cdot \vec{n}_{k}\left(u^{*}\right)
\end{aligned}
$$

As the objective is to find the optimized machining surface so that the envelope surface best fits the set of $B_{k}\left(u^{*}\right)$ points defining the designed surface, the least-square criterion is used, leading to the following optimization scheme:

Find $\overrightarrow{\delta C b_{l}}$ and $\overrightarrow{\delta C t_{m}}$, the displacements of the directrices control points so that $W=\sum_{u^{*}} \sum_{k} e_{k}^{2}\left(u^{*}\right)$ is minimal.

This leads to solve a large linear system, $A \cdot x=b$ with $3 \cdot\left(n_{1}+n_{2}\right)$ equations, where $n_{1}$ and $n_{2}$ are the number of control points of the bottom and the top curve respectively.

First experiments have highlighted that the large linear system is most generally ill-conditioned; solutions are inconsistent [20]. Hence, the Singular Value Decomposition (SVD) must be used to solve the system.

\subsection{Resolution by means of the Singular Value Decomposition}

In order to solve the linear system, the SVD is used leading thus to an estimate of the solution. Indeed, the SVD gives an estimate of a given matrix by a lower rank matrix of same dimensions. The method consists in the decomposition of a rectangular matrix into a product of three matrices, two orthogonal matrices 
and a diagonal matrix:

$$
A=U \cdot \Sigma \cdot V^{T}
$$

with :

- $U \in M_{m \times m}(\mathbb{R})$ and $V \in M_{n \times n}(\mathbb{R})$ are normalized.

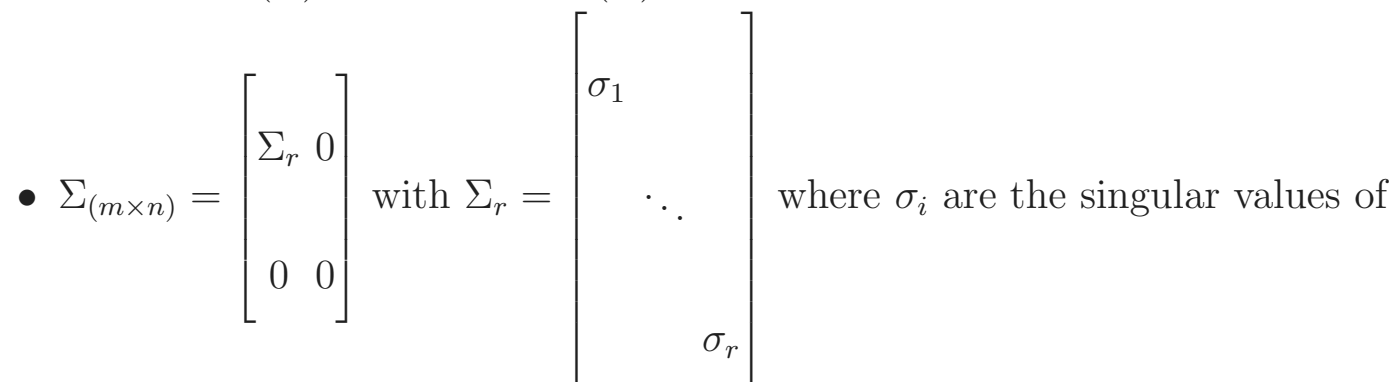
$A$ such as $\sigma_{1}>\cdots>\sigma_{r} \geq 0$. Singular values of a matrix $A \in M_{m \times n}(\mathbb{R})$ are roots of the eigenvalues of $A^{T} \cdot A: \sigma_{i}=\sqrt{\lambda_{i}\left(A^{T} \cdot A\right)}$.

If $A$ is a positive semi-definite matrix, the pseudo-inverse of $A$ is obtained from the eigenvalues $\lambda_{i}$ of $A$ as follows:

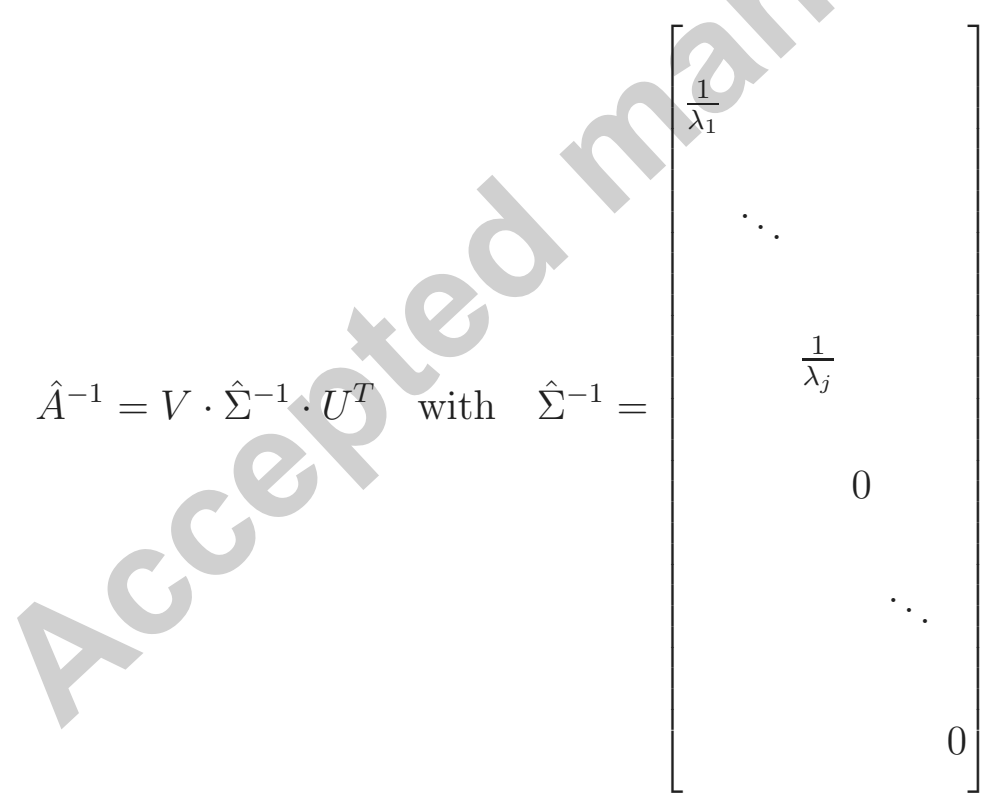

Therefore, the estimate of the solution depends on the rank $j$ of the pseudoinverse matrix (limit value of the eigenvalues). The rank determines the level of approximation. To each $\lambda_{i}$ corresponds a pseudo solution of the system giving 
points define the directrices of the optimized $M S$. The pseudo solution which gives the minimum of $W=\sum_{u^{*}} \sum_{k} e_{k}^{2}\left(u^{*}\right)$ is called the minimal deviations solution.

\section{Minimum energy trajectories}

As mentioned previously, the smoothness of the trajectories is a key criterion for high performance machining within the context of HSM. Machine tool solicitations are decreased for trajectories at least continuous in curvature [21]. Within the framework of curve and surface fittings, many authors use the energy of deformation as a criterion to control element smoothness [22], [23]. The work reported in [24] shows in particular that the energy is the most essential criterion when fitting curves and surfaces to clouds of points. As the tool trajectory is represented as a surface, the evaluation of the smoothness through the energy seems to be relevant. For this purpose, the calculation of the energy of deformation as proposed in [24] is adopted in the present work. The energy of deformation $E$ of a parametric surface $S(u, v)$ on a domain $D$, is given by the following formula:

$$
E=\iint_{D}\left(\left|\frac{\partial^{2} S}{\partial u^{2}}\right|^{2}+2 \cdot\left|\frac{\partial^{2} S}{\partial u \partial v}\right|^{2}+\left|\frac{\partial^{2} S}{\partial v^{2}}\right|^{2}\right) d u d v
$$

Since we are dealing with a ruled parametric surface, this leads to:

$$
E=\iint_{D}\left(\left|\frac{\partial^{2} S}{\partial u^{2}}\right|^{2}+2 \cdot\left|\frac{\partial^{2} S}{\partial u \partial v}\right|^{2}\right) d u d v
$$


To each estimate of the solution, the energy of the $M S$ is calculated using Eq.

10. The objective is thus to find a pseudo solution that gives minimal geometrical deviations while preserving a correct smoothness of the trajectory. This point is investigated through various examples in the next section. Note that the energy along the tool path is numerically calculated using the trapezium rule. The value next denoted Energy corresponds to the cumulated energy for the whole tool path.

\section{Applications}

To show the efficiency of the Geo5XF method, two different kinds of experiments are conducted. First, literature surfaces are tested in order to compare results obtained with Geo5XF to those proposed in literature. The second experimentation concerns the application of the method to an industrial part composed of various patch surfaces. Indeed, literature surfaces are most generally composed of a unique patch surface which simplifies flank milling positioning.

\subsection{Literature surfaces : "Senatore" and "Two flipped" surfaces}

Various surfaces are proposed in literature to compare flank milling positioning algorithms. Among these surfaces, two test surfaces have been selected, both non developable ruled surfaces; the first one is called the "Two flipped" surface as defined in [3] (Fig.4), and the second one is "Senatore" as it was introduced by Senatore in [25] (Fig.4).

For each test surface, minimal and maximal deviations obtained with the 
Geo5XF. In addition, in order to evaluate actual trajectory smoothness, the Energy is calculated and then correlated to the kinematical behaviour during machining. Particular attention is paid to the evolution of the tool axis during machining and to the effective relative tool/surface velocity. Both surfaces are machined using a cylindrical tool with a diameter equal to $20 \mathrm{~mm}$ for the "Two flipped" surface and $25 \mathrm{~mm}$ for the "Senatore" one.

\subsubsection{Geometrical deviations}

Geometrical deviations obtained using the various positioning algorithms are reported in Table 1. Concerning Geo5XF, reported values result from the double projection calculation obtained for the minimal deviations solution. Whatever the surface, Geo5XF gives satisfactory results close to the optimal values obtained with methods issued from literature. Values given for the methods mentioned in Table 1 are taken from the authors' original papers.

\subsubsection{Tool path Smoothness}

To analyze the influence of the Energy, three estimates of the solution of the linear system defined by Eq.(8) are tested. That means three pseudo solutions given for three different eigenvalues: the minimum energy solution (corresponding to min.E), the minimal deviations solution (corresponding to min. $W$ ) and a compromise between energy and deviation, which locally minimizes the energy (corresponding to comp.). In Table 2, maximal and minimal deviations as well as the Energy and the sum of the squared deviations are reported for all three cases. 
For the "Two flipped" surface, results are different according to the considered

pseudo solution. Therefore, the correlation between energy and kinematical behaviour is only investigated for this surface. In order to link the energy to the kinematical behaviour, the evolution of the tool axis orientation in the Part Coordinate System (PCS) is studied, through the evolution of the coordinates $i, j$ and $k$ of the tool axis. As shown in Fig.5, the evolution of $k$ is smoother for the minimum energy trajectory than for the other ones. Furthermore, when zooming, the correlation between the energy and the level of oscillation is clearly highlighted: the greater the energy, the more important the oscillations. Energy thus seems a relevant parameter to characterize trajectory smoothness in the PCS.

In order to check the efficiency of the smoothness criterion in the Machine Coordinate System (MCS), actual machining is performed on a MIKRON UCP 710. The machine tool is a RRTTT structure, and the Inverse Kinematical Transformation (IKT) leads to solve equations below, which have zero, one, two or an infinite number of solutions:

$$
A= \pm \arccos (k) \text { and } C=-\arctan \left(\frac{i}{j}\right) \quad[\pi]
$$

where $A$ and $C$ are the two rotational axes of the machine tool. The previous system of equations has two domains of solutions corresponding to $A>0$ or $A<0$ which vary in function of the coordinates $i, j$ and $k$ of the tool axis.

The IKT is carried out in real time by the NC unit SIEMENS 840D of the machine tool. Machining conditions are as follows: the tool is cylindrical, with a diameter equal to $20 \mathrm{~mm}$; the programmed feedrate is set to $5 \mathrm{~m} / \mathrm{min}$ and the spindle speed to $18000 \mathrm{rev} / \mathrm{min}$. The evolution of machining time is reported in Table 2 for the three pseudo solutions confirming the correlation between 
machining time.

As a partial conclusion, the energy of the $M S$ is a good parameter to evaluate the smoothness of the trajectory in the PCS. For the proposed example, as there is no swapping of solution space during the machining ( $A$ and $C$ keep the same sign during machining), the smoothness is also effective in the MCS. This result has to be confirmed through various examples.

\subsection{Industrial surface: Impeller}

Among papers dealing with flank milling, only a few one have undertaken the issue of industrial multi patch surfaces. In order to emphasize the efficiency of the Geo5XF method, the second illustration proposed concerns an impeller which is characteristic of the complex surfaces industrials attempt to flank mill (Fig.4). The impeller is made of several blades each one defined by compound surfaces. The method is applied to one of the blades leading to a unique trajectory for the machining of both sides of the blade. Table 3 gathers results obtained considering, as in the previous example, three different pseudo-solutions: the minimum energy, the minimal deviations and a compromise. Due to the fact that the surface is representative of industrial complex surfaces, deviations are clearly greater than deviations obtained for literature surfaces. Moreover, as it is essential to obtain at the same time smoothness while minimizing geometrical deviations, the compromise makes sense.

The tool trajectory is calculated according to the following machining conditions: a cylindrical tool with a diameter equal to $6 \mathrm{~mm}$; the programmed 
The evolution of the cumulated energy along the trajectory is reported in Fig.7 for the three estimates of the solution. The figure brings out the adequacy of the energy value to the pseudo-solution; the minimum energy solution gives the minimal cumulated energy. Moreover, for the three trajectories, the gap of energy is clearly visible at the transition edge between the intrados and the extrados where the curvature is important. However, the magnitude of the gap varies according to the pseudo-solution. Hence, a gap in the evolution of the cumulated energy corresponding to the machining of the middle of both the extrados and the intrados only exists for the minimal deviations and the compromise solutions, accounting for trajectories less smooth than with the minimal energy solution.

The evolution of $A$ and $C$ (Fig.8) and (Fig.9) shows that oscillations are more important for the greatest energy pseudo-solution; the smoothest the tool path, the fastest (Table 3). Furthermore, levels are clearly visible in the evolution of $C$ for times belonging to the intervals $[2.4 ; 4.8]$ and $[10 ; 12]$ which seems in adequacy with the energy gaps when machining the intrados and the extrados (Fig.7).

A picture of each machined surface corresponding to each pseudo solution is presented in Fig.10 showing the influence of the energy on the surface finish. Marks at the middle of the extrados are consistent with the energy gaps observed in Fig.7. The lack of marks confirms the smoothness effect of the energy parameter. 
As shown previously, when using the concept of the $M S$, it is possible to minimize geometrical deviations while preserving the trajectory smoothness through the energy of deformation of the $M S$. This statement is specifically valid when there is a single solution when passing from the PCS to the MCS. This is not always the case. Let us consider the surface proposed by Liu (Fig.4) which is machined according to the following conditions: a cylindrical tool with a diameter equal to $20 \mathrm{~mm}$, a programmed feedrate of $5 \mathrm{~m} / \mathrm{min}$ and a spindle speed of $18000 \mathrm{rev} / \mathrm{min}$.

The smoothness analysis shows that the correlation between machining times and energy values has disappeared (Table 4). Machining time for the minimum deviation tool path is shorter than for the compromise whereas its energy is greater.

The evolution of the rotational axis $C$ (Fig.11), brings out that $C$ is positive for the minimum deviations solution, whereas it is negative for the two other pseudo-solutions. Indeed, as the sign of $i$ is not the same at the machining starting point, the rotational axis $C$ moves to the programmed point along the shortest path, through the negative or positive values, according to the NC unit settings. During machining, IKT may involve solution space changes which are not predictable when considering only the energy of deformation in the PCS. 
In this paper, the Geo5XF method - a method designed to optimize tool positioning in 5-axis flank milling - has been detailed. Taking advantage of the surface representation of the tool trajectory, the method leads to globally minimize global gouging between the tool and the surface. Indeed, as far as geometrical deviations are concerned, results obtained with Geo5XF are similar to those obtained in literature. However, in 5-axis High-Speed Machining the control of the trajectory smoothness is essential for high performance trajectory. Hence, as the trajectory is defined as a surface, the smoothness is evaluated through the energy of the surface. Results show that, in general, minimum energy trajectories are the fastest. Energy is thus a relevant parameter to account for the trajectory smoothness. The limit of the method is linked to the IKT, as there is no univocity between the PCS in which the tool trajectory is calculated and the MCS system in which it is executed. Investigating smoothness in the MCS will be a future development of the method.

\section{Acknowledgments}

This work is funded by Missler Software and the French Ministry of Research. 
[1] H. Tonshoff, N. Rackow, Optimal tool positioning for five-axis flank milling of arbitrary shaped surfaces, Annals of the German Academic Society for Production Engineering (WGP) 7 (1) (2000) 57-60.

[2] F. Rehsteiner, H. Renker, Collision free five-axis milling of twisted ruled surfaces, Annals of the CIRP 42 (1) (1993) 457-461.

[3] C. Menzel, S. Bedi, S. Mann, Triple tangent flank milling of ruled surfaces, Computer-Aided Design 36 (3) (2004) 389-296.

[4] W.-W. Liu, Five-axis nc cylindrical milling of sculptured surfaces, Computer-Aided Design 27 (12) (1995) 887-894.

[5] J. Redonnet, W. Rubio, G. Dessein, Side milling of ruled surfaces ; optimum positioning of the milling cutter and calculation of interference, The International Journal of Advanced Manufacturing Technology 14 (7) (1998) 459-465.

[6] F. Monies, J.-M. Redonnet, W. Rubio, P. Lagarrigue, Improved positioning of a conical mill for machining ruled surfaces: application to turbine blades, Proceedings of the Institution of Mechanical Engineers, Part B: Journal of Engineering Manufacture 214 (7) (2000) 625-634.

[7] J. Senatore, F. Monies, J-M.Redonnet, W. Rubio, Improved positioning for side milling of ruled surfaces: Analysis of the rotation axis's influence on machining error, International Journal of Machine Tools and Manufacture 47 (6) (2007) 934-945.

[8] S. Bedi, S. Mann, C. Menzel, Flank milling with flat end milling cutters, Computer-Aided Design 35 (3) (2003) 293-300.

[9] J. Chiou, Accurate tool position for five-axis ruled surface machining by swept envelope approach, Computer-Aided Design 36 (10) (2004) 967- 
[10] H. Gong, L.-X. Cao, J. Liu, Improved positioning of cylindrical cutter for flank milling ruled surfaces, Computer-Aided Design 37 (12) (2005) $1205-1213$.

[11] C. Lartigue, E. Duc, A. Affouard, Tool path deformation in 5-axis milling using envelope surface, Computer-Aided Design 35 (4) (2003) 375-382.

[12] P. Bourdet, L. Mathieu, C. Lartigue, A. Ballu, The concept of small displacement torsor in metrology, Advanced Mathematical Tools in Metrology II, Series on advances in mathematics for applied sciences 40 (1996) $22-100$.

[13] M.-C. Ho, Y.-R. Hwang, C.-H. Hu, Five-axis tool orientation smoothing using quaternion interpolation algorithm, International Journal of Machine Tools and Manufacture 43 (12) (2003) 1259-1267.

[14] S. Lavernhe, C. Tournier, C. Lartigue, Kinematical performance prediction in multi-axis machining for process planning optimization, International Journal of Advanced Manufacturing Technology 37 (5-6) (2008) $534-544$.

[15] A. Affouard, E. Duc, C. Lartigue, J.-M. Langeron, P. Bourdet, Avoiding 5-axis singularities using tool path deformation, International Journal of Machine Tools and Manufacture 44 (4) (2004) 415-425.

[16] L. Piegl, W. Tiller, The Nurbs Book : Second Edition, Springer, 1997.

[17] K. Abdel-Malek, H.-J. Yeh, Geometric representation of the swept volume using jacobian rank-deficiency conditions, Computer-Aided Design 29 (6) (1997) 457-468.

[18] J. Yang, K. Abdel-Malek, Approximate swept volumes of nurbs surfaces or solids, Computer-Aided Design 22 (1) (2005) 1-26.

[19] C. Li, S. Mann, S. Bedi, Error measurements for flank milling, Computer- 
Aided Design 37 (14) (2005) 1459-1468.

[20] W. H. Press, S. A. Teukolsky, W. T. Vetterling, B. P. Flannery, Numerical recipes in $\mathrm{C}$ : The art of scientific comuting, Cambridge University Press, 1992.

[21] A. Dugas, J. Lee, J.-Y. Hascoet, An enhanced machining simulator with error analysis, Journal of Manufacturing Systems 21 (6) (2003) 451-564.

[22] I. Faux, M. Pratt, Computational geometry for design and manufacture, Mathematics and its applications, Ellis Horwood, 1979.

[23] T. Vassilev, Fair interpolation and approximation of b-spline by energy minimization and points insertion, Computer-Aided Design 28 (9) (1996) $753-760$.

[24] X. Wang, F. Cheng, B. Barsky, Energy and b-spline interproximation, Computer-Aided Design 29 (7) (1997) 485-496.

[25] J. Senatore, F. Monies, J.-M. Redonnet, W. Rubio, Analysis of improved positionning in five-axis ruled surface milling using envelope surface, Computer-Aided Design 37 (10) (2005) 989-998. 

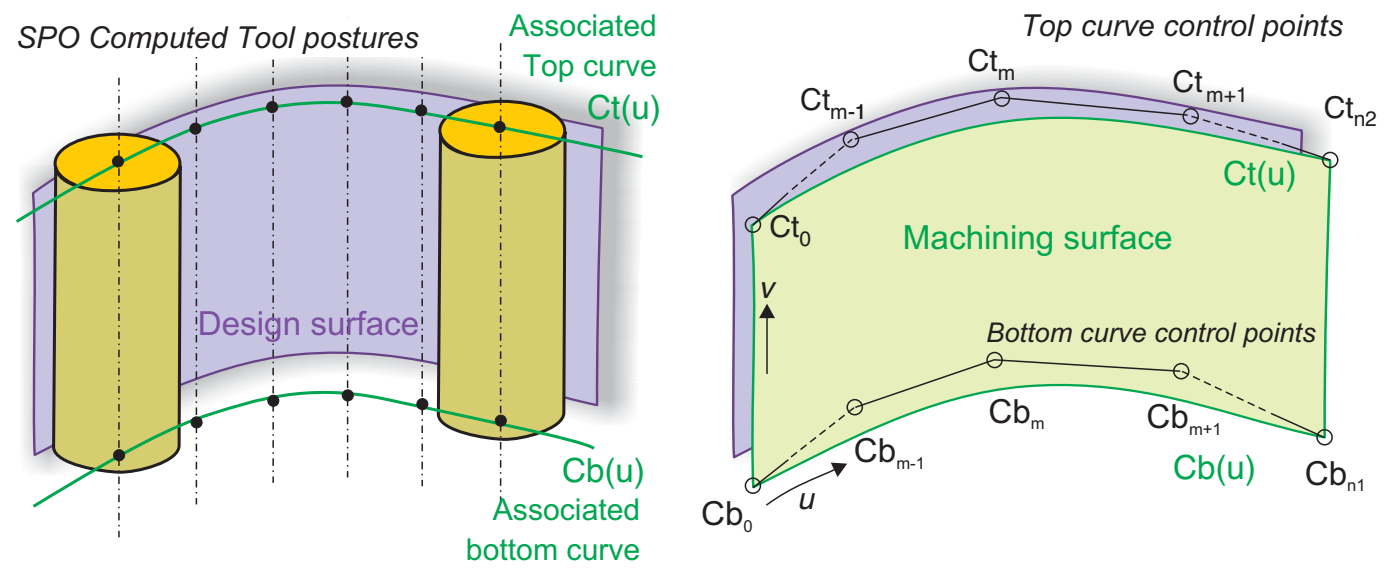

Fig. 1. Geo5XF method: approximation of SPO tool postures (left) and optimisation of the Machining Surface (right) 


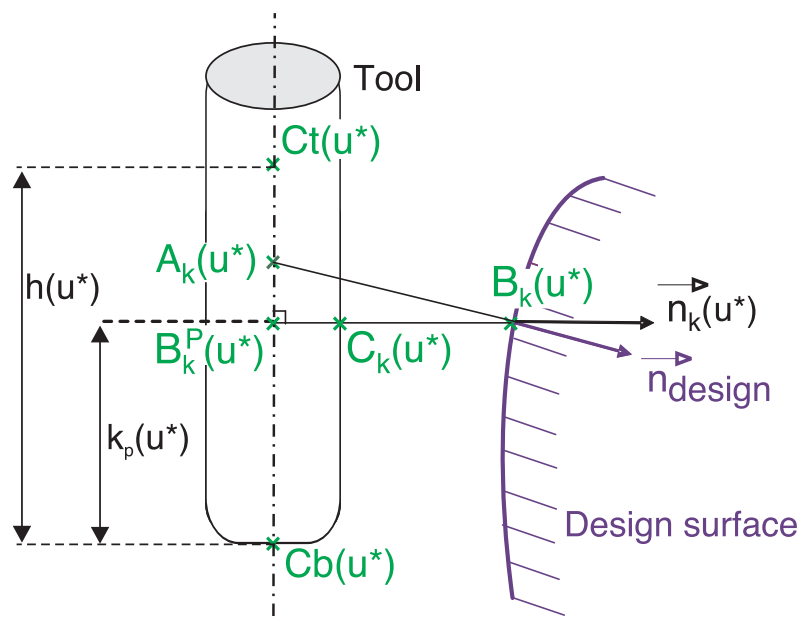

Fig. 2. Double projection to estimate geometrical deviations 


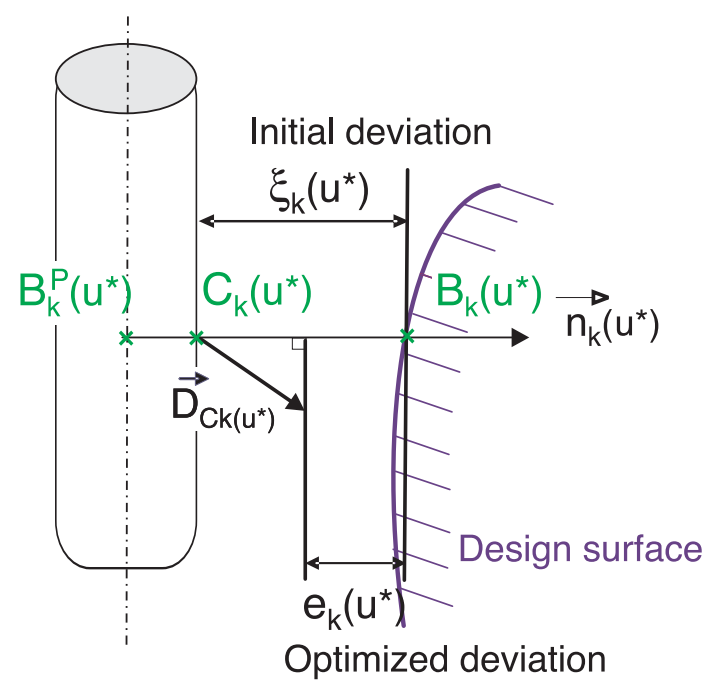

Fig. 3. Computation of optimized deviation 


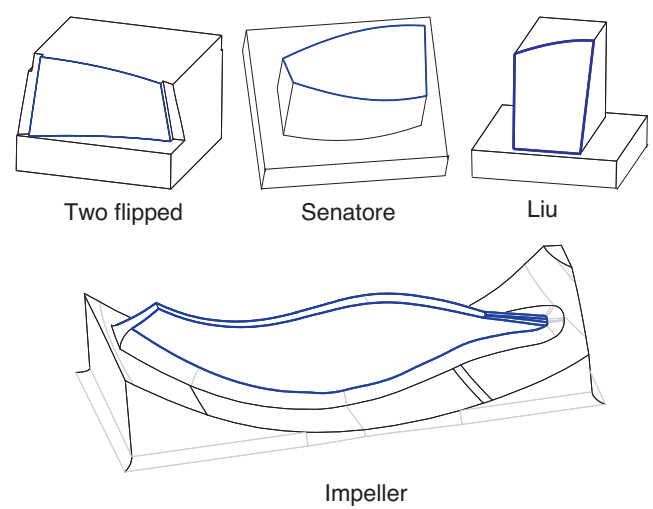

Fig. 4. Test surfaces

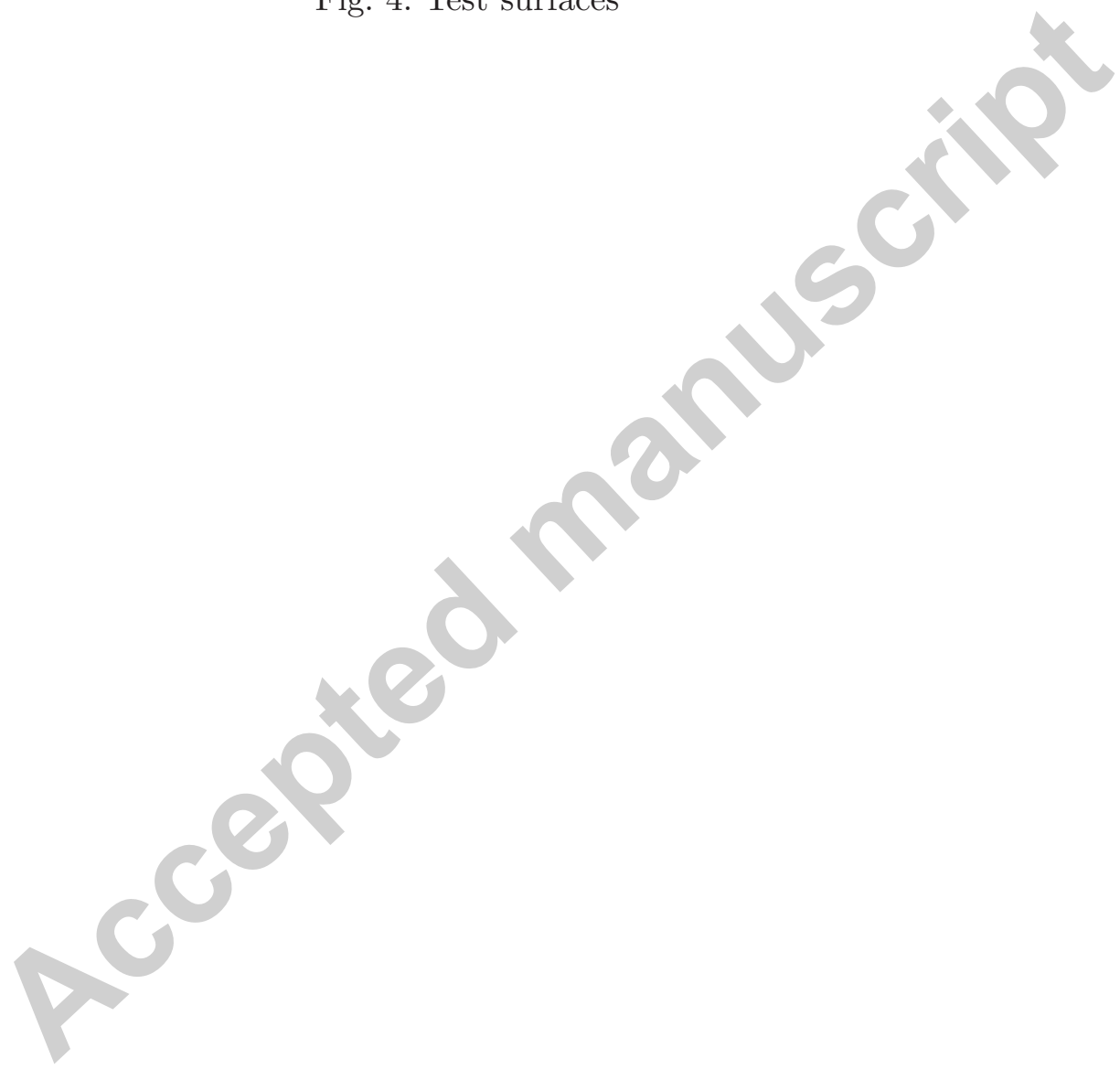




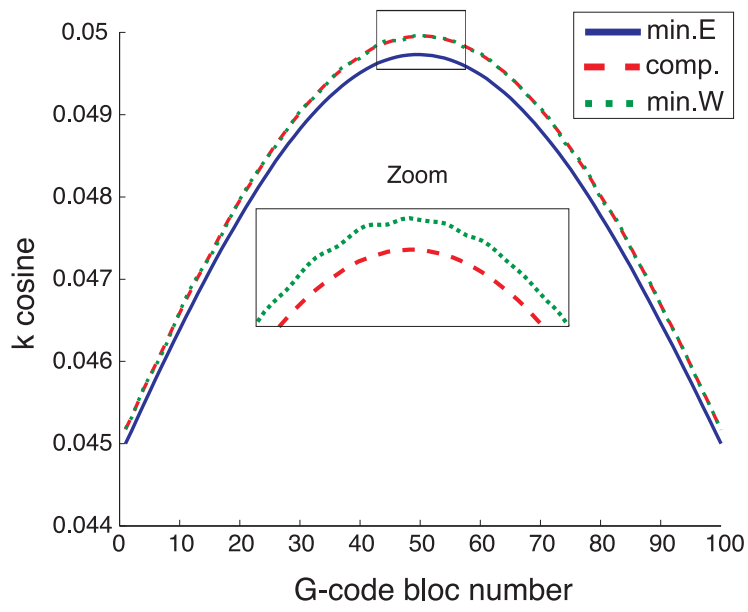

Fig. 5. Evolution of the tool axis orientation (Two flipped surface) 


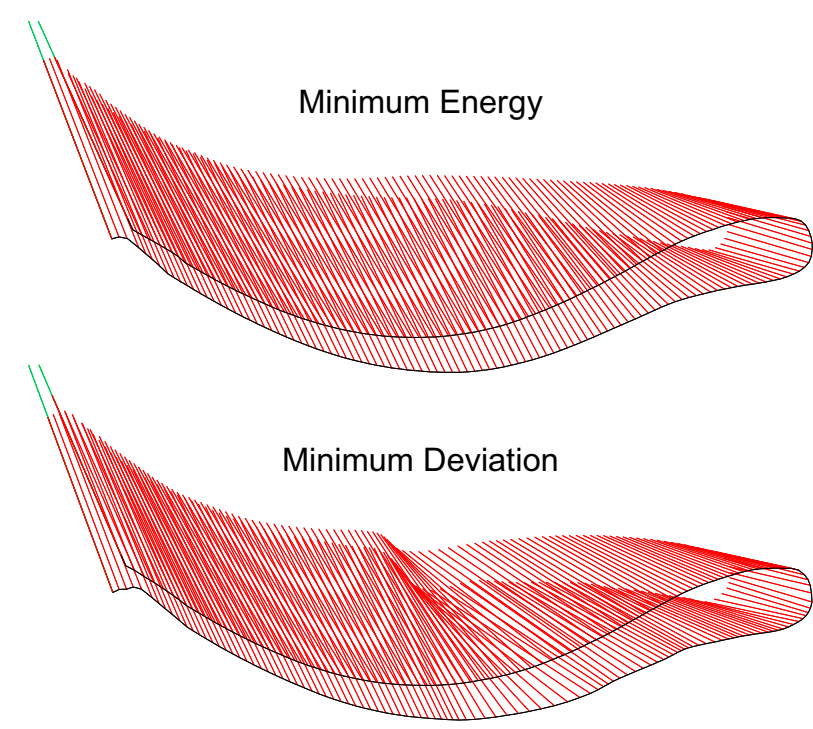

Fig. 6. Computed tool paths (Impeller surface) 


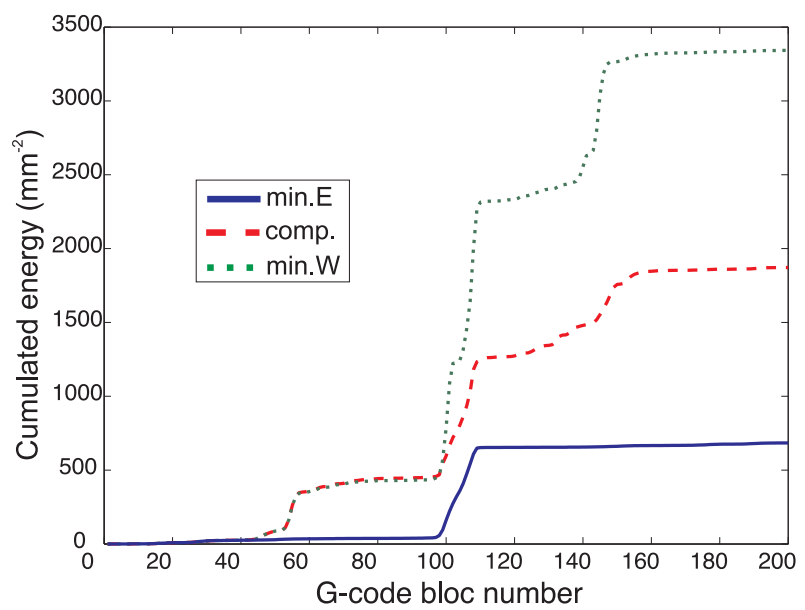

Fig. 7. Cumulated energy of deformation (Impeller surface) 


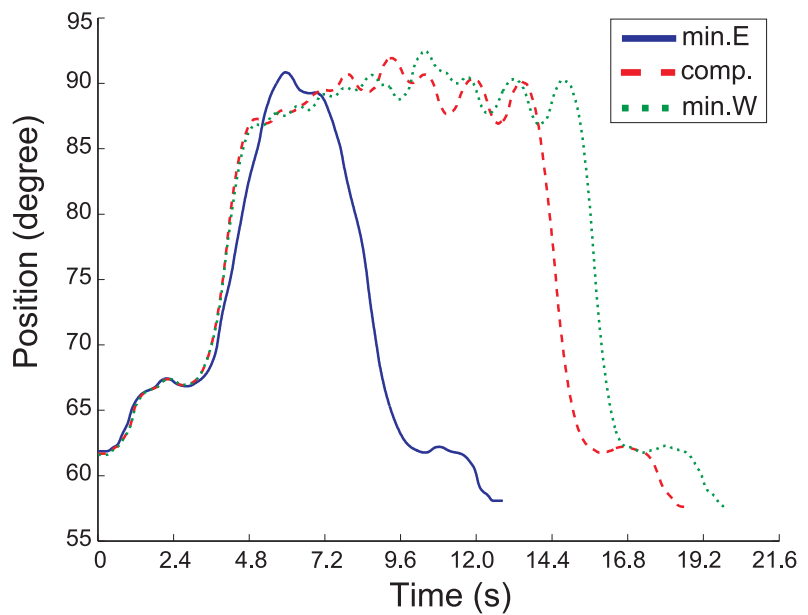

Fig. 8. Evolution of $A$ axis of the machine tool (Impeller surface) 


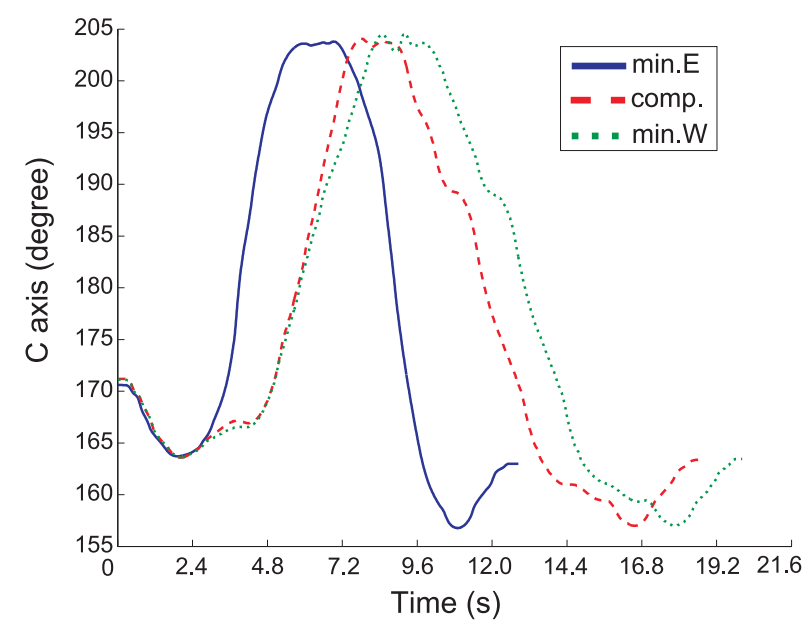

Fig. 9. Evolution of $C$ axis of the machine tool (Impeller surface) 


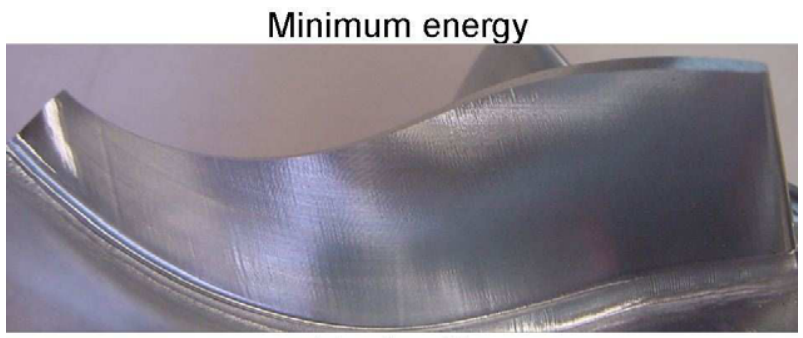

Trade-off

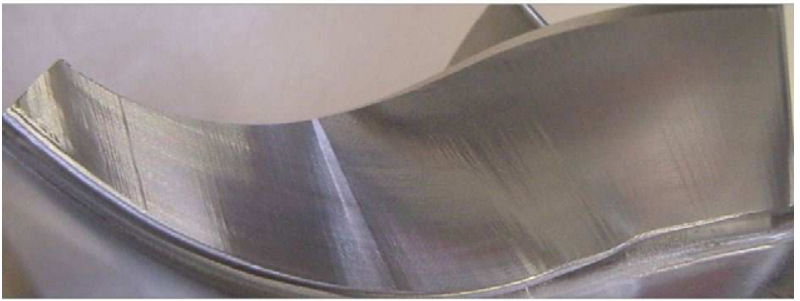

Minimum deviation

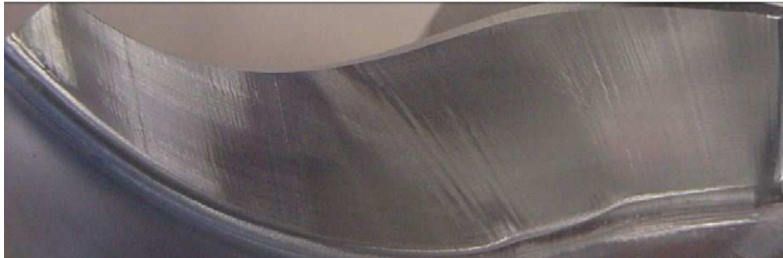

Fig. 10. Effective machined surfaces (Impeller surface) 


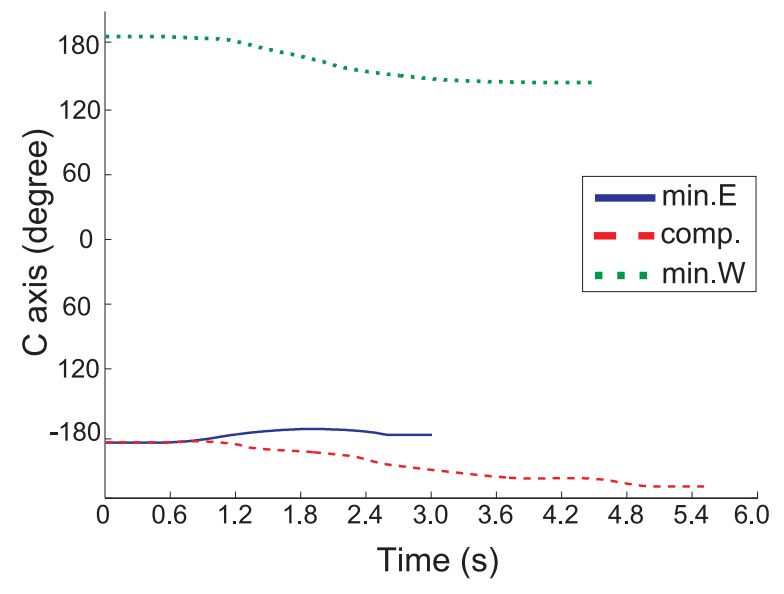

Fig. 11. Evolution of $C$ axis of the machine tool (Liu surface) 
ACCEPTED MANUSCRIPT

\begin{tabular}{|c|c|c|c|c|c|c|}
\hline Test surface & \multicolumn{3}{|c|}{ "Two flipped" } & \multicolumn{3}{c|}{ "Senatore" } \\
\hline Algorithms & Bedi & Menzel & Geo5XF & Redonnet & Senatore & Geo5XF \\
\hline Undercut $(\mathrm{mm})$ & 0.2876 & 0.0061 & 0.0086 & 0 & 0 & 0.007 \\
\hline Overcut $(\mathrm{mm})$ & 0 & 0.0091 & 0.016 & 1.5 & 0.0165 & 0.07 \\
\hline
\end{tabular}

Table 1

Performances of the Geo5XF method in terms of geometrical deviations 


\section{Table 2}

\begin{tabular}{|c|c|c|c|}
\hline \multirow{2}{*}{ Test surface } & \multicolumn{3}{|c|}{ "Two flipped" } \\
\hline Pseudo solution & min.E & comp. & min.W \\
\hline Undercut $(\mathrm{mm})$ & 0.57 & 0.0086 & 0.0086 \\
\hline Overcut $(\mathrm{mm})$ & 0.035 & 0.016 & 0.016 \\
\hline$\sum e_{i}^{2}\left(\mathrm{~mm}^{2}\right)$ & 163 & 0.0470 & 0.0468 \\
\hline Energy $\left(\mathrm{mm}^{-2}\right)$ & 1.60 & 2.22 & 4.68 \\
\hline Time $(\mathrm{s})$ & 4.8 & 5.0 & 5.1 \\
\hline
\end{tabular}

Results of Geo5XF method in terms of geometrical deviations, energy of deformation and machining time (Two flipped surface) 


\section{Table 3}

\begin{tabular}{|c|c|c|c|}
\hline Test surface & \multicolumn{3}{|c|}{ "Impeller" } \\
\hline Pseudo-solution & min.E & comp. & min.W \\
\hline Undercut (mm) & 0.63 & 0.19 & 0.2 \\
\hline Overcut $(\mathrm{mm})$ & 0.8 & 0.42 & 0.39 \\
\hline$\sum e_{i}^{2}\left(\mathrm{~mm}^{2}\right)$ & 208 & 85.2 & 79.3 \\
\hline Energy $\left(\mathrm{mm}^{-2}\right)$ & 686 & 1870 & 3350 \\
\hline Time $(\mathrm{s})$ & 12.5 & 18.5 & 19.8 \\
\hline
\end{tabular}

Results of Geo5XF method in terms of geometrical deviations, energy of deformation and machining time (Impeller surface) 


\section{Table 4}

\begin{tabular}{|c|c|c|c|}
\hline Test surface & \multicolumn{3}{|c|}{ "Liu" } \\
\hline Pseudo-solution & min.E & comp. & min.W \\
\hline$\sum e_{i}^{2}\left(\mathrm{~mm}^{2}\right)$ & 1013 & 10.9 & 7.4 \\
\hline Energy $\left(\mathrm{mm}^{-2}\right)$ & 0.47 & 0.58 & 0.67 \\
\hline Time (s) & 2.6 & 5.0 & 4.0 \\
\hline
\end{tabular}

Results of Geo5XF method in terms of energy of deformation and machining time

(Liu surface) 